\title{
Análisis del rol de la venografía de extremidades inferiores por tomografía computada en pacientes con sospecha clínica de tromboembolismo pulmonar
}

\author{
Dres. Claudio Silva F-A(1), Pablo Soffia $S^{(1)}$, Daniel Pérez $C^{(2)}$, Felipe Vergara $D L^{(3)}$.
}

1. Médico Radiólogo, Departamento de Imágenes. Facultad de Medicina Clínica Alemana, Universidad del Desarrollo Santiago - Chile.

2. Residente postgrado Programa de Especialidad en Radiología Facultad de Medicina Clínica Alemana, Universidad del Desarrollo. Santiago - Chile.

3. Residente postgrado Programa de Especialidad en Radiología Universidad de Santiago. Santiago - Chile.

Analysis of the role of CT Venography of lower limbs in patients with clinically suspected pulmonary embolism

\begin{abstract}
Objectives. To determine the role of CT venography and identify variables that determine the diagnosis of deep venous thrombosis (DVT) of lower extremities in patients with clinical suspicion of PE, and who underwent chest CT angiography. Design. Retrospective group study that evaluated 151 patients in whom lower extremity venography-chest CT angiography was performed. Risk factors were tabulated - such as age, sex, department origin, risk of PE (according to Wells Criteria), previous Doppler ultrasonography of lower extremities and their results, diagnosis and anatomical site of PE, and diagnosis and anatomical site of DVT according to the results obtained with the venography. Results. Complete data was available for 151 patients. 40 were positive for $P E(26.5 \%)$ and 13 venographs were positive (8.6\% of the total). The case origins were $41.7 \%$ from Emergency Department, 31.1\% from ICU, $19.8 \%$ hospitalized (non-critical) and $7.2 \%$ from outpatient consultations. Of the variables studied, none resulted statistically significant for predicting the discovery of deep vein thrombosis in conjunction with CT pulmonary angiography. In those patients with contemporary Doppler ultrasound, the CT venography did not provide new information from that already available. Conclusions. In this study we did not find variables that support a priori the realization of a venography phase in conjunction with chest CT angiogram when there is clinical suspicion of pulmonary embolism. Performing CT venography in patients with stable hemodynamics and suspected PE, would not be justified.
\end{abstract}

Keywords: CT, CT venography, Pulmonary embolism.

Resumen: Objetivos. Determinar el rol de la venografía por tomografía computada e identificar variables que determinen el diagnóstico de trombosis venosa profunda de extremidades inferiores en pacientes con sospecha clínica de TEP y sometidos a angiografía de tórax por tomografía computada. Diseño. Cohorte retrospectiva que evaluó 151 pacientes en los que se realizó venografía de extremidades inferiores - angiografía de tórax mediante tomografía computada. Se tabularon factores de riesgo como la edad, sexo, servicio de proveniencia, riesgo para TEP (según score de Wells), ecotomografía Doppler de EEIl previa y sus resultados, diagnóstico y lugar anatómico de TEP y diagnóstico y lugar anatómico de TVP según los resultados obtenidos con la venografía. Resultados. Se dispuso de información completa para 151 pacientes. 40 fueron positivo para TEP (26,5\%) y 13 venografías resultaron positivas (8.6\% del total). Los casos provenían en un $41,7 \%$ de Urgencia; $31,1 \%$ de UPC; $19,8 \%$ hospitalizados (no crítico); y $7,2 \%$ de consultas ambulatorias. De las variables estudiadas, ninguna resultó estadísticamente significativa para predecir el hallazgo de trombosis venosa profunda en concomitancia con la angiografía pulmonar mediante TC. En aquellos pacientes con ultrasonido Doppler contemporáneo, la venografía por tomografía computada no aportó nueva información de aquella ya disponible. Conclusiones. En este estudio no se encontraron variables que a priori apoyen la realización de una fase venográfica en concomitancia con una angiografía de tórax mediante tomografía computada cuando existe la sospecha clínica de tromboembolismo pulmonar. No se justificaría realizar venografía por TC en pacientes con hemodinamia estable y sospecha de TEP.

Palabras claves: Tomografía computada, Tromboembolismo pulmonar, Venografía por tomografía computada. 
Silva $C$, et al. Análisis del rol de la venografía de extremidades inferiores por tomografía computada en pacientes con sospecha clínica de tromboembolismo pulmonar. Rev Chil Radiol 2014; 20(2): 51-55.

Contacto: Daniel Perez C. / jdperezc@gmail.com

Trabajo recibido el 28 de febrero de 2014. Aceptado para publicación el 08 de junio de 2014.

\section{Introducción}

La enfermedad tromboembólica es una patología frecuente y de gran relevancia clínica. Afecta a 71 personas por cada 10.000 habitantes por año en la población general, aumentando a 960 por cada 10.000 en pacientes hospitalizados ${ }^{(1)}$. La causa de esta patología es multifactorial y se manifiesta principalmente en dos escenarios clínicos: trombosis venosa profunda y tromboembolismo pulmonar; la primera es responsable de alrededor de $2 / 3$ del total, mientras que el TEP del tercio restante. Se asume que más del $90 \%$ de los émbolos pulmonares provienen de las venas de la extremidad inferior y de la pelvis ${ }^{(2,3)}$.

Dentro del estudio imagenológico de ambas entidades, la ecotomografía doppler de extremidades inferiores $(70 \%$ de sensibilidad y $96 \%$ de especificidad) y la angiografía pulmonar mediante tomografía computada (sensibilidad de $83 \%$, especificidad de $96 \%)$ son las técnicas imagenológicas más utilizadas para confirmar la sospecha clínica de TVP y TEP respectivamente ${ }^{(4,5,6)}$

Se ha propuesto que el estudio concomitante de la pelvis y extremidades inferiores (venografía por tomografía computada), mostraría un rendimiento similar a la ecotomografía doppler para el diagnóstico de TVP, simplificando y acortando el estudio de la enfermedad tromboembólica ${ }^{(4,7,8)}$. El examen venoso complementario se realiza cuando el material de contraste inyectado para la angiografía pulmonar circula a través del sistema venoso de la pelvis y extremidades inferiores. De esta forma, no sólo permite conocer el origen más probable del TEP, sino que también pudiera incrementar el diagnóstico de enfermedad tromboembólica si detecta pacientes con TVP en ausencia de TEP(7-9).

Algunos estudios han mostrado que la realización de angio TC de tórax y venografía por tomografía computada tiene una sensibilidad y especificidad mayores para la detección de embolía pulmonar que la angio TC por sí sola en pacientes con riesgo alto de sufrir una enfermedad tromboembólica ${ }^{(5,8,11)}$. A pesar de esto, las diferentes variables que convierten a un paciente en buen candidato para realizar ambas técnicas no han sido escasamente totalmente dilucidadas ${ }^{(11,12,13)}$.

Hay que considerar además que, al incluir el estudio venográfico, la radiación a la cual se expone la pelvis fluctúa entre 3.2 and $9.1 \mathrm{mSv}$, mientras que la dosis recibida por las extremidades inferiores va de 0.6-2.3-mSv ${ }^{(11-14)}$

El objetivo de este trabajo es identificar variables pre-test que justifiquen la realización simultánea de angiografía y venografía por tomografía computada en pacientes con sospecha clínica de TEP.

\section{Material y método}

Estudio de cohorte retrospectiva, a nivel individual, con aprobación del Comité de Ética Institucional, quien autoriza exención de consentimiento informado.

La población objetivo fueron pacientes con sospecha clínica de TEP provenientes del Servicio de Urgencias, Unidad de Pacientes Críticos (UPC), Hospitalizados (no-críticos) y pacientes ambulatorios de Clínica Alemana de Santiago durante un período de 12 meses. En estos pacientes se realizó una angioTAC en conjunto con una fase venográfica de extremidades inferiores. Los exámenes fueron adquiridos con los siguientes parámetros: $120 \mathrm{kV}$, $220 \mathrm{mAs}$; tiempo de rotación de 0,5s, pitch de 1 a 1,2, colimación de $128 \times 0,6$. Se administraron 70-100 ml de contraste yodado no-iónico iv (Omnipaque 350 \&) a un flujo 4-5 ml/seg, seguido de $50 \mathrm{ml}$ de solución salina isotónica (bolus chaser), a través de teflón $18 \mathrm{G}$ ubicado en vena antecubital o acceso venoso disponible. Mediante técnica de "Bolus Tracking" con $\mathrm{ROI}$ en arteria pulmonar y umbral de $100 \mathrm{UH}$, se inició la adquisición a los 5 segundos aproximadamente de alcanzado el umbral. A los 3 min de inyectado, se realizó barrido venográfico de extremidades inferiores hasta las fosas poplíteas.

Fueron estudiadas las siguientes variables:

1. Sexo

2. Edad

3. Servicio de procedencia: Urgencia, sala básica, intermedio y UCl

4. Riesgo para TEP (según score de Wells): Alto o bajo

5. Ecotomografía doppler de EEIl: previa (48hrs), diagnóstico y lugar anatómico TVP

6. AngioTAC: Diagnóstico y lugar anatómico TEP

7. Venografía: Diagnóstico y lugar anatómico TVP

\section{Método estadístico}

Análisis descriptivo de la población, en conjunto con las variables predictoras del estudio. Posteriormente, se realizó una regresión logística multivariable de los predictores para explorar posibles interacciones que aumenten la probabilidad del resultado positivo venográfico. Se estimaron intervalos de confianza de $95 \%$, cuando pertinente, y se consideró un valor significativo $p<0.05$. Todos los análisis se realizaron con software STATA 10.0 (STATA ${ }^{\circledR}$, Stata Corp LP).

\section{Resultados}

La población en estudio fue de 151 pacientes. 50.3\% del total fueron mujeres y un $49.7 \%$ hombres. Respecto a la edad, la mediana fue de 67 años, con un rango in- 
terquartil de 30 años (rango: 19-95 años) (Figura 1). La distribución del servicio de procedencia fue 63 pacientes del Servicio de Urgencias (44.6\%); 47 paciente de Unidades de Pacientes Críticos-UPC (31.1\%); 30 pacientes de Hospitalizados (19.8\%); y 11 pacientes $(7.2 \%)$ de consultas ambulatorias (Figura 2). Respecto al riesgo para TEP de la población, categorizado según score de Wells, un $47 \%$ poseía riego bajo y un $53 \%$ riesgo alto.

Respecto al diagnóstico de TEP, la angio tomografía demostró ser positivo en 40 pacientes (26.5\%). De los cuales 16 pacientes pertenecían a urgencia (40\%); 15 pacientes a sala básica (37.5\%); 5 pacientes a UPC (12,5\%); y 3 pacientes ambulatorios (7,5\%) (Figura 2). En un $87,5 \%$ la ubicación del trombo fue lobar.

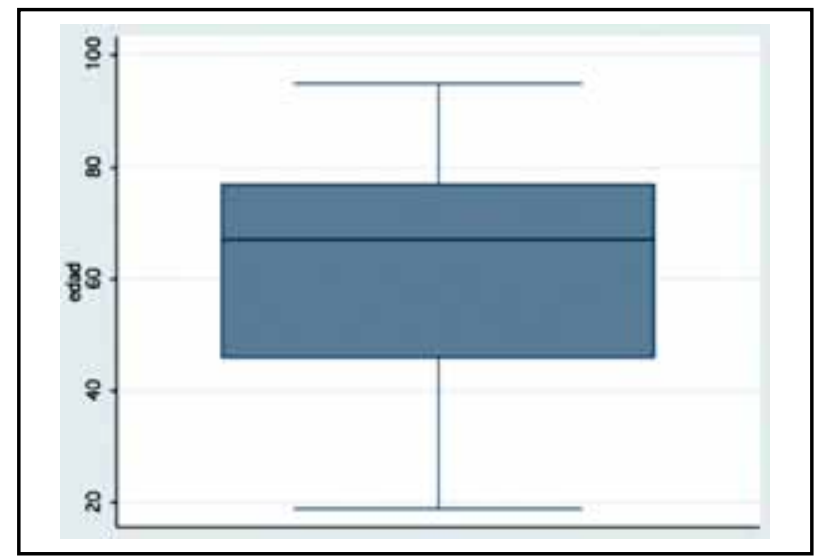

Figura 1. Distribución etaria de la población estudiada, con una mediana de 67 años.

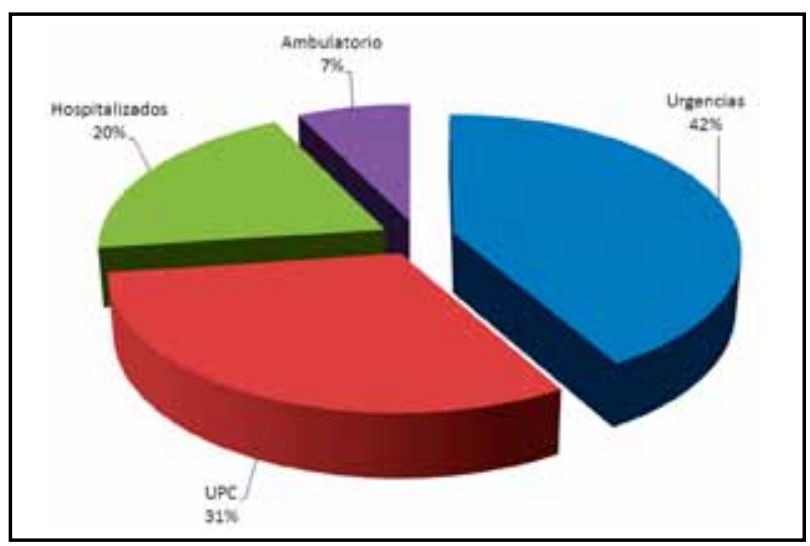

Figura 2. Distribución de servicio de procedencia de la población estudiada. Destaca el Servicio de Urgencias con un $44.6 \%$ y UPC con $31.1 \%$.

La ecotomografía previa fue realizada en 21 pacientes, once de los cuales fueron positivos para TVP $(52,3 \%)$; ocho de éstos fueron también positivos para TEP. El lugar anatómico más frecuente fue la vena femoral superficial y el territorio infrapoplíteo.

La venografía por TC fue positiva para TVP en 13 pacientes (8.6\% del total), nueve de los cuales fueron positivos para TEP (Figuras 3 y 4). En aquellos con venografía positiva sin TEP concomitante, tres tenían ultrasonido que habría revelado la TVP. Es decir, sólo en $0.66 \%$ de la muestra la venografía aportó información clínicamente relevante y no conocida de TVP.

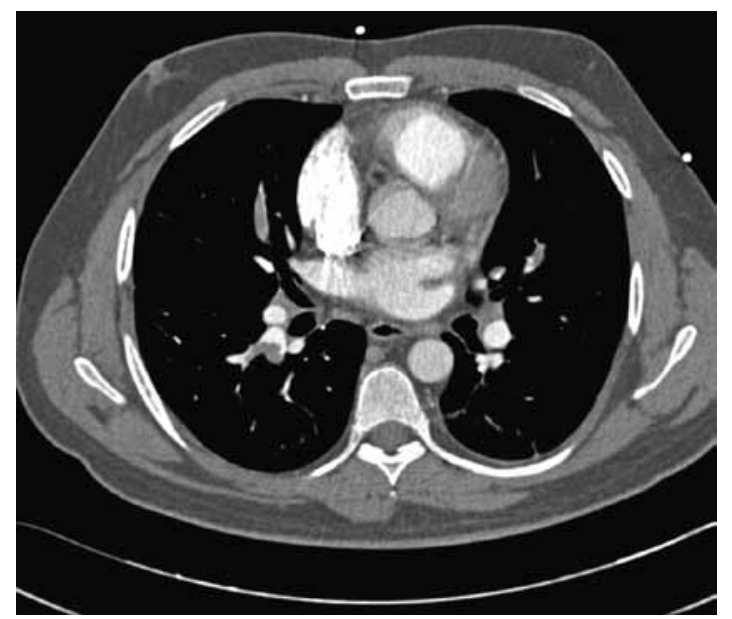

Figura 3. Corte axial de angioTAC de arterias pulmonares que revela defectos de llene en ramas segmentarias y subgmentarias.

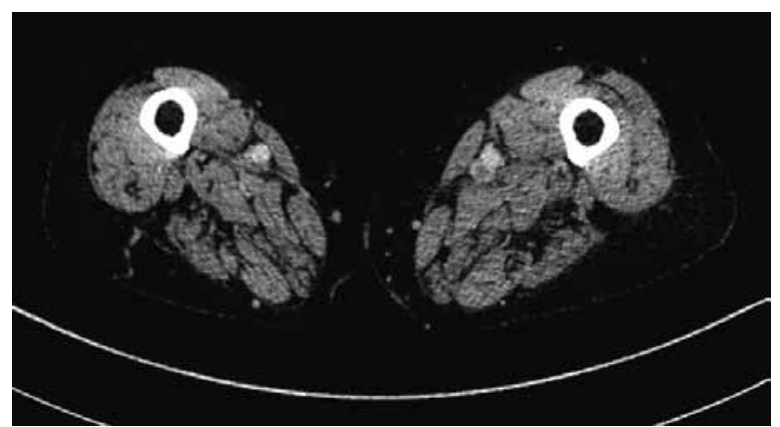

Figura 4. Del mismo paciente, es un corte venográfico del tercio medio del muslo, con defecto de llene en vena femoral superficial izquierda. En este paciente, el estudio previo con ecografía Doppler había revelado la trombosis venosa profunda.

La regresión logística multivariable no mostró valores significativos para las variables edad, sexo, riesgo y resultado de la angio TC. Sin embargo, en el análisis univariado, destacó el resultado de la angioTC (+) (Tabla I), la que evidenció un odds ratio de $7.7(p=0.001)$; en otras palabras, esto significa que es 7.7 veces más probable encontrar un resultado venográfico positivo si se encuentra un resultado de angioTAC positivo.

Tabla I. Resultado del análisis univariado de factores predictores de venografía positiva para trombosis venosa profunda, con sus valores de Odds Ratio, intervalos de confianza de $95 \%$ y valor-p.

\begin{tabular}{|c|c|c|c|}
\hline & OR & IC 95\% & $P$ \\
\hline Edad & 1 & $0,9-1$ & 0,6 \\
\hline Sexo & 0,8 & $0,2-2$ & 0,7 \\
\hline Servicio & 0,8 & $0,5-1,3$ & 0,6 \\
\hline Riesgo & 0,4 & $0,1-1,6$ & 0,2 \\
\hline AngioTC (+) & $\mathbf{7 , 7}$ & $\mathbf{2 , 2 - 2 6}$ & $\mathbf{0 , 0 0 1}$ \\
\hline
\end{tabular}




\section{Discusión}

En nuestro estudio la población no presentó grandes diferencias demográficas, con un perfil etario representativo de la población adulta, con mínimo de 19 y un máximo de 95 años. Con respecto al género, la división fue equitativa, con prácticamente un $50 \%$ de representatividad para cada género.

Respecto al origen de los pacientes según el servicio de procedencia, un $42 \%$ provino del servicio de urgencias; un $38 \%$ de sala básica; $12,8 \%$ de UPC; y $7,6 \%$ de atenciones ambulatorias.

Nuestros resultados revelan que la adición de venografía por tomografía computada sólo aportó un diagnóstico de TVP $(0.66 \%)$ que no era conocido por estudios previos y que determinaría un cambio terapeútico como uso de anticoagulantes orales. En este caso correspondió a un paciente de UPC, probablemente de mayor dificultad diagnóstica.

Este estudio va en concordancia con estudios recientes que plantean una reformulación del uso de venografía por tomografía computada en el contexto del estudio del tromboembolismo pulmonar, en particular en el escenario de disponer de ultrasonido para la evaluación de las venas de las extremidades inferiores $(7,17,18)$.

$\mathrm{Si}$ consideramos que la dosis efectiva para la realización de venografía es de aproximadamente $1.48 \mathrm{mSv}$ para hombres y $1.68 \mathrm{mSv}$ para mujeres, nos enfrentamos al hecho de agregar radiación a la región gonadal por un aporte marginal $(0.66 \%)$. Una consideración aparte radica en los pacientes críticos, donde sus características clínicas pudieran limitar la realización de la ecografía Doppler de extremidades inferiores, y considerando que su hemodinamia puede ser más impredecible, pudiera ser razonable plantear su realización en casos seleccionados.

Como limitante de este estudio podemos mencionar que dado el carácter retrospectivo de este estudio, nos impide estudiar concordancia formal entre venografía y ultrasonido Doppler, debido a que sólo se realizaron 21 ecotomografías, a diferencia de las 151 venografías. Sin embargo, está demostrado que el rendimiento de ambas técnicas es comparable ${ }^{(4)}$.

Consideramos que en atención a los datos obtenidos, venografía por tomografía computada debiera eliminarse del estudio estándar de angiografía por tomografía computada ante sospecha por TEP en pacientes hemodinámicamente estables.

\section{Conclusión}

En este estudio no se encontraron variables que a priori apoyen la realización de una fase venográfica en concomitancia con una angioTC de tórax cuando existe la sospecha clínica de un tromboembolismo pulmonar. Sólo existe una mayor probabilidad de un resultado venográfico positivo cuando el angioTAC es positivo para TEP, hallazgo irrelevante clínicamente.

\section{Bibliografía}

1. Heit JA, Melton LJ 3rd, Lohse CM. Incidence of venous thromboembolism in hospitalized patients vs community residents. Mayo Clin Proc 2001 Nov; 76(11): 1102-1110.

2. White $\mathrm{RH}$. The epidemiology of venous thromboembolism. Circulation 2003; 107(23 Suppl 1): 14-18.

3. Hyers TM. Venous thromboembolism. Am J Respir Crit Care Med 1999; 159:1-14.

4. Goodman LR, Stein PD, Matta F. CT venography and compression sonography are diagnostically equivalent: data from PIOPED II. AJR Am J Roentgenol 2007 Nov; 189(5): 1071-1076.

5. Garg K, Kemp JL, Wojcik D, Hoehn S, Johnston RJ. Thromboembolic Disease: CT Comparison of Combined CT Pulmonary Angiography and Venography with Bilateral Leg Sonography in 70 Patients. AJR 2000; 175: 997-1001.

6. Stein PD, Fowler SE. Multidetector computed tomography for acute pulmonary embolism. N Engl J Med 2006 Jun 1; 354(22): 2317-2327.

7. Kalva SP, Jagannathan JP, Hahn PF, Wicky ST. Venous Thromboembolism: Indirect CT Venography during CT Pulmonary Angiograph Should the Pelvis Be Imaged? Radiology 2008; 246 (2): 605-611.

8. Bierry G, Holl G, Kellner F, Riehm S, Roedlich MN, Greget $M$, et al. Venous thromboembolism and Occult Malignancy: Simultaneous Detection During Pulmonary CT Angiography with CT Venography. AJR 2008; 191: 885-889.

9. Andresen M, González A, Díaz O. Evaluación prospectiva de la venografía mediante angioTC en el diagnóstico de enfermedad tromboembólica. Rev Med Chile 2010; 138: 1480-1486.

10. Tapson VF. Acute pulmonary embolism. N Engl J Med 2008; 358: 1037-1052.

11. Goodman LR, Stein PD, Beemath A. CT venography for deep venous thrombosis: continuous images versus reformatted discontinuous images using PIOPED II data. AJR Am J Roentgenol 2007; 189(2): 409-412.

12. Cham MD, Yankelevitz DF. Thromboembolic disease detection at indirect CT venography versus CT pulmonary angiography. Radiology 2005; 234: 591-594.

13. Rademaker J, Griesshaber V, Hidajat N. Combined CT pulmonary angiography and venography for diagnosis of pulmonary embolism and deep vein thrombosis: radiation dose. J Thorac Imaging 2001 oct; 16(4): 297-299.

14. Hurwitz LM, Reiman RE, Yoshizumi TT. Radiation dose from contemporary cardiothoracic multidetector CT protocols with an anthropomorphic female phantom: implications for cancer induction. Epub 2007 Oct 8. Radiology 2007 Dec; 245(3): 742-750.

15. Nazaroglu H, Özmen CA, Akay HO. 64-MDCT Pulmonary Angiography and CT Venography in the Diagnosis of Thromboembolic Disease. AJR 2009; 192: 654-661.

16. Hunsaker A, Zou K, Poh A. Routine Pelvic and Lower Extremity CT Venography in Patients Undergoing Pulmonary CT Angiography. AJR 2008; 190: 322-326.

17. Slater $S$, Oswal $D$, Bhartia B. A retrospective study of the value of indirect $C T$ venography: a British perspective. $\mathrm{Br}$ J Radiol 2012; 85: 917-920.

18. Goodman LR, Sostman HD, Stein PD, Woodard PK. CT Venography: A Necessary Adjunct to CT Pulmonary Angiography or a Waste of Time, Money, and Radiation? Radiology 2009; 250: 327-330. 